\title{
El desarrollo de la planta docente propuesto por la RIEMS
}

\author{
The development of the teaching staff \\ proposed by the RIEMS
}

Francisco Guzmán Marin, ${ }^{*}$ Gabriela Díaz Orozco y Gabriela Berenice Soto Moreno ${ }^{* *}$

\section{RESUMEN}

La investigación que sustenta el presente artículo comprende tres dimensiones: primero, el análisis hermenéutico de los documentos de la RIEMS; segundo, la contrastación crítica entre la oferta de capacitación docente para la EMS, las competencias docentes y las necesidades de los profesores de este nivel educativo $y$, tercero, la ponderación, también crítica, del desarrollo de las prácticas educativas en tres centros escolares, pertenecientes a distintos subsistemas del bachillerato en el estado de Michoacán. El presente constituye parte de un primer informe de investigación más amplio y expone los primeros resultados del análisis hermenéutico de los documentos que fundamentan la aplicación institucional de la RIEMS, así como de las políticas generales de la formación continua de los profesores para este nivel educativo, las implicaciones profesionales que comportan las ocho competencias docentes y su relación con las once implicadas en el perfil de egreso que propone la misma reforma educativa.

Palabras clave: RIEMS, educación media superior, formación docente, educación, competencias

\section{ABSTRACT}

The research underlies this article includes three dimensions: first, the hermeneutic analysis of the documents of the RIEMS; second, the critical testing between the supply of training for EMS, teaching competences and specific need of teachers of this educational level; and third, weighting, also critical, of the real development of educational practices in three different schools, from different subsystems of high school in the state of Michoacán. These articles is a part of a first report broader research and present the first results of hermeneutical analysis of the documents underlying the institutional implementation of RIEMS, as well as the general policies of the continuous training of teachers for this level education and the professional implications that involve the eight teaching competences and their relationship with eleven competencies of the graduate profile that proposes the same educational reform.

Key words: RIEMS, high school, teacher training, education, competences

* Profesor-Investigador de la Unidad 161, Morelia, de la UPN; coraxthelastone@hotmail.com

** Maestrantes en Educación Media Superior de la Unidad 161, de la UPN, en Morelia, Mich. 


\section{PRESENTACIÓN GENERAL}

Tradicionalmente, la formación de profesores [EMS] ha quedado implícita en el requerimiento de contar con una formación general profesional comprendida en los programas de licenciatura afines a las materias a ser impartidas $y$ los cursos que cada institución organiza para sus profesores Armando Alcántara / Juan Fidel Zorrilla

A prácticamente una década de la publicación del Acuerdo 442, por el cual se determina el establecimiento del Sistema Nacional del Bachillerato en un Marco de Diversidad (SNB) y de los acuerdos correspondientes que posibilitan la instauración del enfoque educativo por competencias en las prácticas formativas del nivel de preparatoria (Acuerdos 444 y 447), luego de la instauración de la Reforma Integral de la Educación Media Superior (RIEMS) en México, resulta pertinente revisar, crítica y sistemáticamente, cómo y en qué medida las acciones de actualización y capacitación docente han propiciado la transformación de las prácticas educativas, en la inteligencia de que la RIEMS, dentro del marco del mejoramiento de los procesos formativos, tiene como antecedente directo la Reforma Curricular el Bachillerato General, impulsada por la Dirección General de este nivel educativo en los ciclos escolares 2003-2004 y 2006-2007, cuyo propósito fue la materialización de la estrategia de impartir una "Educación Media Superior de buena calidad" (SEP, 2013b), considerada en el Programa Nacional de Educación 2001-2006, donde se anticipa el cambio significativo del modelo educativo vigente, conforme a las tendencias mundiales predominantes en el ámbito de la educación, hacia la imposición del enfoque educativo por competencias.

La investigación comprende tres dimensiones: por un lado, el análisis hermenéutico de los documentos básicos de la RIEMS, con la finalidad de identificar con precisión los alcances formales del mejoramiento de las competencias docentes y su relación con las exigencias formativas del nuevo modelo educativo; por otro, la contrastación crítica entre la oferta de capacitación y actualización docente para la Educación Media Superior (EMS), las competencias docentes propuestas por la propia RIEMS y las necesidades concretas de los profesores de este nivel educativo; y 
por último, la ponderación, también crítica, del desarrollo real de las prácticas educativas en tres diferentes centros escolares, pertenecientes a distintos subsistemas del bachillerato, en el estado de Michoacán. El presente artículo expone los primeros resultados del análisis hermenéutico de los documentos que fundamentan la aplicación institucional de la RIEMS, así como de las políticas generales de la formación continua de los profesores para este nivel educativo y las implicaciones profesionales que comportan las ocho competencias docentes y su relación con las once competencias del perfil de egreso que propone la misma reforma educativa.

En principio, conviene advertir la emergente confluencia de tres fenómenos socioeducativos determina que la Educación Secundaria y la EMS se conviertan en los niveles estratégicos y prioritarios del Sistema Educativo de México en las últimas tres décadas: en primera instancia, la gradual inversión de la pirámide poblacional que posiciona a los pubertos, adolescentes y jóvenes adultos, esto es, a las personas de entre 10 a 20 años de edad, como el sector mayoritario de la sociedad mexicana y, por tanto, grupo etario donde se concentra, actualmente, la mayor demanda de los servicios educativos -rango de edad que comprende la formación del Bachillerato- (ver gráfica 1); en segunda, la ampliación sistemática de la cobertura de atención en los distintos niveles de la Educación Básica y el paulatino incremento de la escolaridad promedio de la sociedad mexicana redunda en el aumento sostenido de los posibles aspirantes a los diferentes tipos de oferta y modalidades formativas del nivel de preparatoria (la tasa de absorción en el ciclo escolar 2006-2007 asciende a 98.3\% de los egresados de la Educación Secundaria -SEP, 2008b-, mientras que para 2013 se atiende a alrededor de 4.4 millones de adolescentes -de acuerdo con el Diagnóstico Inicial que presenta la Dirección General de Evaluación de Políticas -SEP, 2015: 3-); $y$, en tercera instancia, los acuerdos mundiales, internacionales y regionales en materia de educación, impulsados por los diferentes organismos de coordinación multilateral en el orden de la sociedad global, como la Organización de las Naciones Unidas para la Educación, la Ciencia y la Cultura (UNESCO) y la Organización para la Cooperación y el Desarrollo Económico (OCDE), entre 
otros -así, por ejemplo, la Conferencia Regional de la Educación Superior en América Latina y el Caribe, realizada en Cartagena de Indias, Colombia, en junio del 2008, en relación con la Cobertura y Modelos Educativos e Institucionales que, en su Declaración doceava plantea la indispensabilidad de garantizar la universalización de la educación media (CRES, 2009: 95).

GRÁFICA 1. Pirámide Poblacional 2015

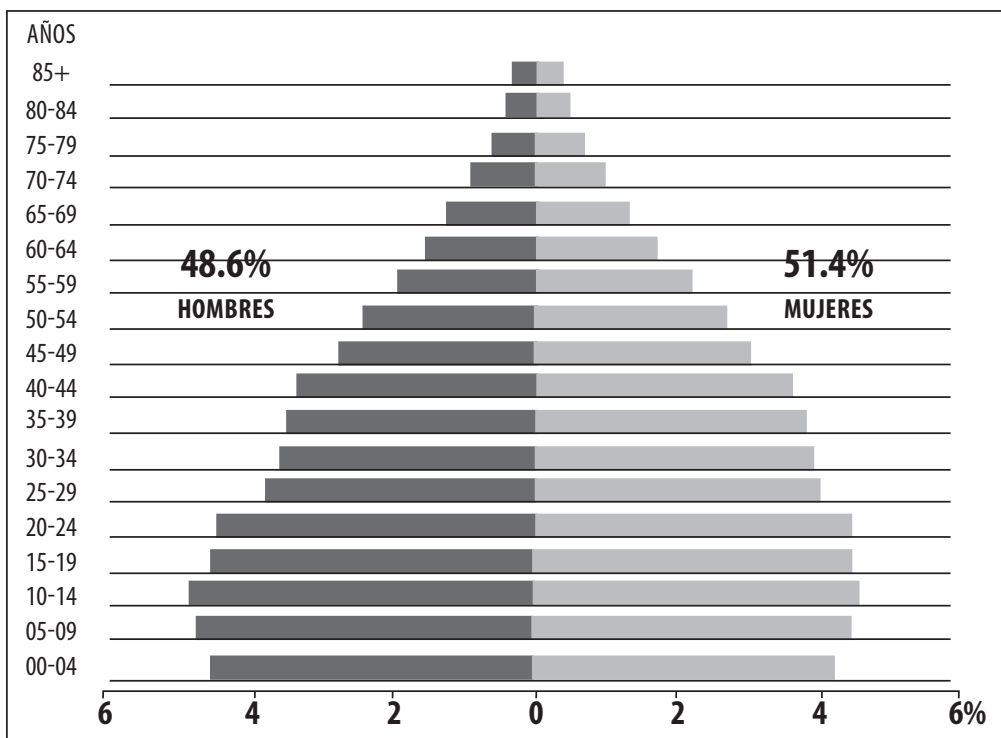

Fuente: Instituto Nacional de Estadística y Geografía. Encuesta Intercensal, 2015.

Los antecedentes directos de la RIEMS, como respuesta estratégica del gobierno federal de México al emergente reposicionamiento de este nivel en el sistema educativo nacional, en principio, se encuentran en el Plan Nacional de Desarrollo 2007-2012 que, en su Eje rector 3. Igualdad de oportunidades, dentro del ámbito del Desarrollo integral, reconoce como un problema nodal "la desvinculación entre la educación media superior y superior y el sistema productivo" (Poder Ejecutivo Federal, 2007: 177), mientras que en su Objetivo 9. Elevar la calidad educativa, Estrategias 9.1 y 9.3 plantean la conformación de "un sistema de evaluación 
integral a cargo de Comités técnicos de expertos en educación” y el rediseño de "los planes de estudio para que los alumnos cuenten con un mínimo de las capacidades requeridas en este nivel (EMS), y les permita transitar de una modalidad a otra" (Poder Ejecutivo Federal, 2007: 183 y 185), de manera respectiva.

Las estrategias propuestas en el PND 2007-2012 pretendieron ser operadas a través del Programa Sectorial de Educación 2007-2012; de esta forma, en su Objetivo 1. Elevar la calidad de la educación para que los estudiantes mejoren su nivel de logro educativo, cuenten con medios para tener acceso a un mayor bienestar y contribuyan al desarrollo nacional, propone las siguientes estrategias para la EMS: 1.6 respecto de la integración del Sistema Nacional de Bachillerato en un marco de respeto a la diversidad de modelos; 1.7 referente al establecimiento de las competencias para la vida y el trabajo comunes y mínimas para la obtención de la certificación nacional de la EMS; 1.8 la definición del perfil docente deseable a fin de orientar las acciones de actualización y capacitación para los profesores de este nivel educativo; 1.9 el establecimiento de un programa de titulación para el personal docente; y 1.10 la instauración de los mecanismos y lineamientos necesarios para la certificación de las competencias docentes que posibiliten la conformación de una planta académica de calidad. Estas metas son complementadas con el Objetivo 2. Ampliar las oportunidades educativas para reducir desigualdades entre grupos sociales, cerrar brechas e impulsar la equidad, que propone como indicadores sustantivos: por un lado, ampliar la cobertura de la EMS a 68\% en 2012 y, por otro, pasar de 15 a 18 entidades federativas la cobertura de este mismo nivel educativo, en al menos 25\%, hacia el mismo año. En estos aspectos del Programa Sectorial se proyectan las principales acciones de la RIEMS, a saber: la constitución del Sistema Nacional de Bachillerato (SNB), la construcción del Marco Curricular Común (MCC) con base en el enfoque educativo por competencias, la profesionalización y certificación de los profesores de la EMS, además de la definición expresa de las distintas modalidades de la oferta educativa en el bachillerato.

En lo que respecta a la planta docente de la EMS, es pertinente señalar que dispone de un insuficiente perfil profesional. 
En términos generales, la planta académica del nivel, en 2006, se caracteriza por los siguientes fenómenos: alrededor de la tercera parte de los profesores carece del grado de licenciatura (28.7\%), mientras que en 2009, en el acto de entrega de los documentos de certificación a la primera generación de docentes, el Subsecretario de EMS, Miguel Székely Pardo, indica que solo 60\% de los maestros del nivel se encontraba titulado, porcentaje que se incrementa a 79.1\% hacia el ciclo escolar 2011-2012, de acuerdo con estimaciones del INEE (2011); de la misma forma, se carece de un programa sistemático de formación inicial, capacitación, actualización y superación profesional para el desarrollo de las competencias docentes que demandan las prácticas educativas de la EMS; mientras que es patente la inexistencia de procesos institucionales claros y precisos para la inserción, promoción, estímulo y certificación de la práctica profesional docente en este nivel educativo.

En efecto, las autoridades de las diversas instituciones del bachillerato confiaron en que la formación y la experiencia profesional en el campo, así como las eventuales acciones de capacitación didáctico-pedagógica y los posibles estudios de postgrado, propiciarían el desarrollo de las competencias docentes; sin embargo, es importante hacer notar que un alto porcentaje de profesores se incorporan a la docencia en la EMS recién egresados de la licenciatura, sin haber desarrollado ninguna experiencia profesional en su campo; otros más se encuentran desempeñándose en áreas ajenas a su profesión y todavía resulta mínimo el porcentaje de profesores con estudios de postgrado - menos de 20\%, al decir de Ibarra Uribe y sus colaboradores (s/f)-. A más de estos fenómenos, resulta conveniente señalar el tipo de nombramiento que persiste en la EMS, en el ciclo escolar 2006-2007, esto es: $16.6 \%$ de tiempo completo, $12.6 \%$ de medio tiempo y $62.3 \%$ de asignación por horas (tendencias que muestran un ligero repunte hacia el ciclo escolar 2011-2012, a saber: 17\%, 14.3\% y 58.6\%, respectivamente), conforme a los datos proporcionados por Ana Esperanza Carreño y sus colaboradores (2013), lo cual implica que la mayoría de los profesores se sustraiga de las ocasionales acciones de formación docente, los trabajos de academia y la planeación institucional, entre otros. El conjunto de estos hechos impacta, de forma significativa, en la baja calidad de los servicios 
educativos del nivel, que suelen reconocer los diversos investigadores en la materia y los diferentes diagnósticos realizados por las distintas dependencias de gobierno y de los organismos multilaterales -como la SEP, la Cámara de Diputados, el Instituto Nacional de Evaluación Educativa (INEE) y la OCDE, entre otros.

\section{LA REFORMA INTEGRAL DE LA EDUCACIÓN MEDIA SUPERIOR}

En el reconocimiento de la compleja problemática que afronta la EMS, tras la histórica apatía de las autoridades federales, y los difíciles retos institucionales que comporta la reorientación estratégica de las diversas experiencias de formación construidas por los distintos subsistemas que conforman al nivel, el impulso a la elevación de la calidad de sus servicios educativos y la emergente articulación con la Educación Superior y los sectores productivos del país, en el entorno de un orden global que demanda una preparación técnico-científico-disciplinaria más sólida y de una mayor participación comprometida de los ciudadanos del mundo, ${ }^{1}$ la SEP consulta e integra diferentes equipos de especialistas en materia de educación, autoridades educativas de los estados de la República, profesores de la Red de Bachilleratos de la Asociación Nacional de Universidades e Instituciones de Educación Superior (ANUIES), expertos de la Universidad Nacional Autónoma de México (UNAM) y del Consejo de Especialistas de la propia Secretaría, con el propósito manifiesto de diseñar una propuesta general que posibilite la transformación significativa de este nivel educativo.

Así, en una primera etapa participan cinco grupos regionales, representantes de las autoridades educativas estatales, en noviembre de 2007; al propio tiempo, durante los meses de septiembre a noviembre del mismo año, se organizaron varias reuniones con especialistas de la Red nacional del nivel medio superior de la ANUIES. En una segunda etapa, durante diciembre del mismo año, se realiza una jornada de trabajo con dos representantes de cada una de las cinco regiones de las entidades federativas, tres

\footnotetext{
"La participación de México en un mundo globalizado guarda estrecha relación con una EMS en expansión, la cual debe preparar a un mayor número de jóvenes y dotarles de las condiciones que el marco internacional exige" (SEP, 2008b: 11).
} 
representantes de la Red y el Equipo técnico especializado de la SEP, para proyectar la versión final de la propuesta de la RIEMS.

Los retos expresos que encara esta propuesta de reforma son:

1. Ampliación de la cobertura. Los problemas de cobertura, más que en la capacidad de absorción de los egresados de la Educación Secundaria, puesto que esta alcanza tasas sostenibles mayores que 98\% a partir del ciclo escolar 2005-2006, se concentran en los altos grados de deserción escolar y de la baja eficiencia terminal, producto de la falta de pertinencia de los estudios frente a las realidades de los contextos locales y regionales del país, así como a las necesidades, intereses y prospectivas de vida de los estudiantes, sin soslayar la baja calidad educativa del nivel y la falta de equivalencias entre los programas de estudio que ofertan los distintos planteles y subsistemas, de acuerdo con las estimaciones de la SEP (2008b), en virtud de lo cual resulta necesario impulsar transformaciones curriculares que flexibilicen la rigidez de los planes y programas de estudio, permitan la movilidad institucional de los alumnos y presenten alternativas concretas de desarrollo personal, a fin de que opten por la permanencia en los centros escolares. Adicionalmente, deben generarse estrategias de compensación académica que solventen las deficiencias y rezagos propiciados en los niveles educativos precedentes, con el propósito de reducir los indicadores de reprobación y la consecuente deserción escolar.

2. Mejoramiento de la calidad. El mejoramiento de la calidad educativa se determina por tres principales factores, estos son: la pertinencia, la calidad de la enseñanza y la actualidad de las instalaciones y el equipamiento del que disponen las instituciones del nivel. La pertinencia se entiende como la correspondencia de los contenidos de formación con los retos que comportan las transformaciones sociopolíticas y económicoculturales de las últimas décadas, así como los dispositivos de generación e intercambio de información, sin omitir las necesidades psicosociales de los adolescentes del presente. La calidad de la enseñanza depende de la definición del perfil profesional docente para la especificidad de la EMS, la imple- 
mentación de los procesos de formación correspondiente para los profesores que se desempeñan en el nivel y la instauración de mecanismos institucionales para asegurar que, quienes se incorporen, ya dispongan de tal perfil. Por su parte, la actualidad de las instalaciones y el equipamiento institucional, redunda en el grado de la calidad de los procesos educativos, particularmente en la formación técnica. A estos tres factores es necesario sumar los procesos de orientación vocacional, las tutorías y la evaluación de los aprendizajes, mediante el establecimiento de estándares comunes a todos los subsistemas que comprende la EMS, con el objeto de formular recomendaciones pertinentes para reforzar fortalezas y atender debilidades del nivel (SEP, 2008b: 14).

3. Búsqueda de la equidad. En la perspectiva de que la educación desempeña una función determinante en la construcción de la equidad nacional, a consecuencia de que representa un presunto factor de movilidad social, parece conveniente convertir a las escuelas de la EMS en un "igualador de oportunidades" que revierta las tendencias de deserción concentradas en los sectores sociales más vulnerables.

En función del reconocimiento de las complejas problemáticas, los profundos retos y las tendencias nacionales e internacionales de reforma a la EMS, así como de las exigencias del desarrollo socioeconómico del país y los rasgos característicos de la población adolescente, en el contexto sociohistórico actual, la SEP (2008b) define tres principios básicos para el diseño e instrumentación de la RIEMS, a saber:

a) Reconocimiento universal de todas las modalidades y subsistemas del Bachillerato. Establecimiento de los consensos interinstitucionales y socioeconómicos necesarios con el objeto de definir un "núcleo irreductible de competencias comunes" para todos los estudiantes de la EMS, sin importar el tipo, modalidad e institución en que se formen, a partir del cual se oriente la sinergia de constitución del SNB, cuya certificación oficial permita el acceso a la Educación superior o al mercado laboral, según la decisión personal de cada egresado. 
b) Pertinencia y relevancia de los planes de estudio. Los planes de estudio de la EMS deben asumir como premisa fundamental responder a las necesidades personales, sociales y laborales de los adolescentes del siglo XXI, en el entorno de la comunidad global, caracterizada por la dinámica de la sociedad del conocimiento, las rápidas transformaciones científico-tecnológicas, la inmediata transferencia de información y la creciente pluralidad sociocultural. Los procesos formativos deben responder a las condiciones psicosociales de los adolescentes, ya sea que aspiren a continuar sus estudios en el nivel superior, o si precisan incorporarse de manera inmediata al mercado laboral.

c) Tránsito entre subsistemas y escuelas. A fin de combatir el problema de la deserción escolar y posibilitar la movilidad estudiantil entre regiones, subsistemas, planteles o modalidades, conforme a sus condiciones específicas de vida y a sus intereses de desarrollo personal, resulta indispensable establecer los dispositivos institucionales necesarios para la portabilidad de los estudios, sin que ello signifique la renuncia a la identidad particular, la filosofía educativa o la experiencia formativa construida por las instituciones que participan en la EMS.

A consecuencia del reconocimiento institucional de este complejo conjunto de factores y elementos socioeducativos se determinan los ejes de la RIEMS, a saber:

1. El Marco curricular común (MCC). Este se organiza en función de tres principios básicos: los Desempeños terminales, el Enfoque por competencias y la Flexibilidad y Componentes comunes.

Los principios básicos, el enfoque por competencias y el propio MCC representan el núcleo fundamental de la estrategia global de la SEP, para la ulterior conformación del SNB que se propone la RIEMS. Ahora bien, ante las diversas alternativas de alcanzar la integración estratégica de los diferentes planes, programas y modalidades de estudio que persisten en la EMS -entre las que se encuentran: los "desempeños finales compartidos", el 
"tronco común" y la definición de un conjunto de "asignaturas afines obligatorias"-, sin renunciar a la diversidad que le es característica, ni forzar una cierta homogeneidad que provocaría mayores resistencias en la comunidad socioeducativa del nivel y dilapidaría la experiencia formativa construida por cada plantel y subsistema, la SEP opta por los denominados desempeńos finales compartidos, dada su viabilidad y conveniencia.

La ventaja fundamental del esquema de los desempeños terminales o finales compartidos es que no requiere de una reestructuración completa de los diversos diseños curriculares propuestos por los distintos subsistemas e instituciones del nivel -disciplinas, asignaturas, objetivos de aprendizaje, temarios, unidades didácticas, módulos, etc.-, sino que solo demanda de una estrategia de articulación formativa cuyo propósito central es conseguir el desarrollo del perfil de egreso, que en este caso se organiza por once competencias genéricas, según propone el Artículo 4, del Acuerdo Número 444 por el que se establecen las Competencias que constituyen el Marco curricular común del Sistema Nacional de Bachillerato. La intención es conciliar el respeto a la diversidad educativa con el logro de objetivos comunes de formación que permitan la certificación oficial del bachillerato. Grosso modo, las principales características de las competencias genéricas son:

ESQUEMA 1. Características de las Competencias genéricas

Competencias Genéricas $\left\{\begin{array}{l}\text { Clave: Aplicables en contextos } \\ \text { personales, sociales, académicos y } \\ \text { laborales amplios. Relevantes a lo } \\ \text { largo de la vida. } \\ \text { Transversales: Relevantes a todas } \\ \text { las disciplinas académicas, así como } \\ \text { a las actividades extracurriculares } \\ \text { y procesos escolares de apoyo a los } \\ \text { estudiantes. } \\ \text { Transferibles: Refuerzan la } \\ \text { capacidad de los estudiantes de } \\ \text { adquirir otras competencias. }\end{array}\right.$

Fuente: SEP, 2008b.

El Acuerdo 444, en su Artículo 2, define las competencias que deben comprobar los desempeños terminales compartidos del MCC, tales son: 


\section{CUADRO 1. Competencias del MCC}

\begin{tabular}{|c|c|c|}
\hline \multicolumn{2}{|c|}{ Competencias } & Objetivo \\
\hline \multicolumn{2}{|l|}{ Genéricas } & $\begin{array}{l}\text { Comunes a todos los egresados de la EMS. Son competencias clave por su importancia } \\
\text { y aplicaciones diversas a lo largo de la vida; transversales, por ser relevantes a } \\
\text { todas las disciplinas y espacios curriculares de la EMS, y transferibles, por reforzar la } \\
\text { capacidad de los estudiantes de adquirir otras competencias. }\end{array}$ \\
\hline \multirow[b]{2}{*}{ Disciplinares } & Básicas & $\begin{array}{l}\text { Comunes a todos los egresados de la EMS. Representan la base común de la formación } \\
\text { disciplinar en el marco del SNB. }\end{array}$ \\
\hline & Extendidas & $\begin{array}{l}\text { No serán compartidas por todos los egresados de la EMS. Dan especificidad al modelo } \\
\text { educativo de los distintos subsistemas de la EMS. Son de mayor profundidad o } \\
\text { amplitud que las competencias disciplinares básicas. }\end{array}$ \\
\hline \multirow[b]{2}{*}{ Profesionales } & Básicas & Proporcionan a los jóvenes formación elemental para el trabajo. \\
\hline & Extendidas & $\begin{array}{l}\text { Preparan a los jóvenes con una calificación de nivel técnico para incorporarse al } \\
\text { ejercicio profesional. }\end{array}$ \\
\hline
\end{tabular}

Fuente: SEP, 2008c.

2. Definición y regulación de las modalidades en oferta. El marco de diversidad en que se pretende la constitución del SNB pretende establecer una identidad compartida a las distintas opciones formativas de la EMS, allende las modalidades específicas en que se oferten, a fin de establecer condiciones institucionales suficientes para disponer del reconocimiento institucional y de la certificación correspondiente, siempre y cuando alcancen determinados estándares mínimos de competencia.

3. Los mecanismos de gestión de la reforma. El propósito de elevar la calidad educativa, disminuir los índices de deserción escolar e instaurar de manera eficiente, en consecuencia, la propia RIEMS requiere de la implementación de ciertos mecanismos de gestión institucional, desde donde se definen estándares y dispositivos procedimentales comunes que pueden hacer posible la universalidad de la EMS, así como el logro de los desempeños terminales compartidos. La reforma considera seis mecanismos de gestión, los cuales se describen a continuación: a) Orientación, tutoría y atención a las necesidades de los alumnos; b) Desarrollo de la planta docente; c) Instalaciones y equipamiento; d) Profesionalización de la gestión; e) Evaluación integral; y f) Mecanismos para el tránsito entre subsistemas y escuelas. 
4. La certificación del Sistema Nacional de Bachillerato. La certificación del SNB complementaria a la expedida por los diversos subsistemas, modalidades y planteles, representa el reconocimiento nacional de que la instancia oferente ha concretado, de forma exitosa, los tres procesos de instauración de la RIEMS, a saber: el desarrollo de las competencias del desempeño terminal compartido que conforman el perfil de egreso de la EMS, la verificación de que la institución ha cumplido con los estándares mínimos requeridos y la consecuente instauración de los mecanismos de gestión que implica la reforma.

En México, la EMS ha padecido, históricamente, descuido institucional, si no es que franco olvido por parte del gobierno federal, a causa de los siguientes factores, de acuerdo con Gustavo Adolfo Ibarra Mercado (2011: 40): la "falta de visibilidad política”; los bajos índices de retención de su población estudiantil; su inexistencia jurídica en el marco constitucional -por lo menos hasta la reforma del párrafo primero, inciso c) de la fracción II y la fracción $\mathrm{V}$ del Artículo $3^{\circ}$ y la Fracción I del Artículo 31 de la Constitución Política de los Estados Unidos Mexicanos, que la convierten en un nivel educativo obligatorio, junto con la Educación Básica, en 2012, así como su correspondiente definición y caracterización en la Ley General de Educación (LGE)-; el inevitable impacto de las ya recurrentes y crónicas crisis socioeconómicas; la continua omisión de la especificidad del nivel en la conceptuación filosófica, psicológica, sociológica y didácticopedagógica por parte de los especialistas en materia de educación; el habitual desinterés social y gubernamental hacia la compleja problemática psicosocial que viven los adolescentes; la función de simple intermediación formativa que desempeña en el Sistema Educativo Nacional -bien propedéutica a la Educación Superior, bien preparatoria para la inserción en el mercado laboral-; y la crisis de identidad que provoca su amplia diversidad institucional, modalidades formativas y tipos de atención, lo cual explica que la SEP solo atienda, de forma directa, alrededor de la tercera parte de la matrícula pública del bachillerato, en el país -un cuarto del total, considerando al sector privado-. La SEMS se crea hasta 2005, con el propósito de coordinar los bachilleratos fe- 
derales. En efecto, por lo general, el interés y la preocupación de la sociedad, los especialistas y las autoridades educativas se concentran en los procesos de desarrollo de la Educación Básica y de la Educación Superior; de hecho, existe una vasta literatura sobre formación infantil y en torno de la teoría andragógica para la educación de los adultos, pero todavía resultan incipientes e insuficientes las propuestas para la formación de los adolescentes.

\section{EL DESARROLLO DE LA PLANTA DOCENTE EN EL MARCO DE LA RIEMS}

En términos de la RIEMS, algunos de los principales factores que propician la deserción escolar en el bachillerato son: la falta de pertinencia de los procesos formativos, la baja calidad del servicio educativo, la falta de atención específica a las deficiencias académicas con que llegan los estudiantes al nivel, los índices de reprobación y la ausencia de incentivos para retener a los alumnos en la continuidad de sus estudios; los cuales, de una u otra manera, se resuelven en la concreción de las prácticas docentes de la institución. Si bien es cierto que la pertinencia de los estudios de la preparatoria se determina desde el diseño curricular, la calidad del servicio depende de un conjunto diverso de condiciones institucionales (entre las que se encuentran la infraestructura, los dispositivos de apoyo académico, la conectividad, las herramientas tecnológicas, los recursos didáctico-pedagógicos, etc.), la atención a las áreas de oportunidad académica de los alumnos y la incentivación pedagógica de los mismos deben ser atendidas desde diversas instancias -los Consejos Técnicos, las Tutorías, las Academias y la Orientación educativa, entre otros-; también es verdad que las prácticas docentes representan el espacio nuclear donde se compensan o se potencian las disfunciones de todas las dimensiones del centro escolar, la modalidad formativa y el subsistema educativo. La práctica docente constituye la caja de resonancia de las decisiones educativas, las dinámicas institucionales y las condiciones instrumentales de formación. Y en el centro de las prácticas educativas se encuentra la formación profesional de la que dispone el profesor. 
En este marco, la profesionalización docente de los profesores del bachillerato, en cuanto mecanismo de gestión de la RIEMS, resulta un proceso estratégico no solo para la consecución específica de los objetivos propuestos por este proyecto educativo del gobierno federal, sino que también representa un factor emergente para contribuir en la elevación de la calidad educativa en el nivel superior y del avance socioeconómico local y regional de México. En efecto, si los egresados del bachillerato arriban con un desarrollo de competencias académicas más sólido a la educación superior, la formación profesional podrá ser más consistente; pero, a su vez, este hecho constituye un agente sustantivo de potenciación económica. Las competencias, en cualquier ámbito de la vida humana, no se reducen a un cierto mecanicismo técnico, acrítico e irreflexivo, funcional a las reiterativas tareas mecánicas del desempeño instrumental en el mercado de trabajo -colocando a los maestros "ante la falsa disyuntiva entre desarrollar competencias o promover la apropiación significativa de contenidos", según afirma un amplio grupo de investigadores del Centro de Investigación y de Estudios Avanzados (CINVESTAV) (Ezpeleta, 2008)-, como pretenden los detractores de este enfoque educativo; por el contrario, debido a su carácter ontointegrador e interdisciplinario, cualquier competencia básica involucra al conjunto de las distintas facultades humanas. De hecho, la especulativa reflexión filosófica y el análisis crítico de las humanidades se sustentan en el desarrollo de determinadas competencias intelectivas; el aprendizaje significativo, por su parte, en términos ontológicos, no significa un proceso opuesto al desarrollo de competencias, razón por la cual, su relación formativa no es disyuntiva sino integradora. El conocimiento, en sí mismo, representa un dominio de competencias.

Ahora bien, el proyecto de la RIEMS dispone, para el fortalecimiento profesional docente de los profesores del bachillerato, de tres mecanismos principales de operación, estos son: por un lado, un marco legal-administrativo que define los rasgos de desempeño del perfil docente, así como el ingreso, promoción, reconocimiento y permanencia en el servicio, con el Acuerdo número 447 por el que se establecen las competencias docentes para quienes impartan educación media superior en la modalidad escolarizada 
y la Ley General del Servicio Profesional Docente (LGSPD); por otro lado, un Programa de Formación Docente de Educación Media Superior (ProForDEMS) de carácter interinstitucional y, por último, un dispositivo institucional bifronte para la evaluación del desempeño docente y la certificación del presunto dominio de las competencias docentes, con el INEE y el Proceso de Certificación de Competencias Docentes para la Educación Media Superior (CERTIDEMS). Adicionalmente, a fin de cerrar el cerco de coacción institucional para que los profesores del bachillerato desempeñen la función que les ha sido asignada por el proyecto de reforma, bajo el legítimo propósito de promover una educación de calidad, la SEP determina los lineamientos del Perfil, Parámetros e Indicadores (PPI) para Docentes y Técnicos Docentes en Educación Superior, que sirven de soporte para decidir la idoneidad de los maestros que participan en este nivel educativo; no hay, pues, opción posible para los profesores, puesto que están obligados a cumplir con el perfil establecido, según se establece en el Artículo 12 de la LGSPD y es convalidado por los Lineamientos para llevar a cabo la Evaluación del desempeño del personal docente y de quienes ejerzan funciones de Dirección y Supervisión en Educación Básica y Media Superior en el Ciclo Escolar 2016-2017 (LINEE) del INEE.

Por lo que se refiere al contenido profesional docente, la RIEMS considera cinco aspectos fundamentales, a saber: el dominio particular de la materia que imparte en el bachillerato, las disposiciones magisteriales propias del enfoque por competencias, los conocimientos de las condiciones psicosociales de los adolescentes, los procedimientos de evaluación educativa y el uso de las TIC en la educación, además de un cierto dominio del diseño curricular que les posibilite participar en la toma de decisiones de la reforma e integrar en la concreción de sus prácticas docentes el desarrollo de las once competencias que constituyen los desempeños terminales compartidos del perfil de egreso del MCC. A estos aspectos, conviene advertir, sin duda alguna, les hacen falta tres elementos centrales en la educación contemporánea del siglo XXI, tales son: por una parte, el reconocimiento de las condiciones sociopolíticas y económico-culturales que posibiliten la contextuación de sus prácticas formativas; por otro 
lado, la identificación de los factores transversales de la integración socioeducativa (el respeto a la diversidad, la conformación de la ciudadanía global, el desarrollo sustentable, entre otros); y por último, las capacidades de autogestión formativa. Un aspecto que debiera ser prioritario en la profesionalización de los docentes de la EMS, si es que se pretende la congruencia educativa con los objetivos institucionales de la reforma, es el desarrollo de las ocho competencias que definen el perfil profesional docente de los profesores, conforme se establece en el Acuerdo 447. Sin embargo, parece prevalecer en la perspectiva de los promotores de la RIEMS, una cierta comprensión instrumentalista del fortalecimiento de la planta docente, al reducir sus alcances al simple propósito de eficientar la aplicación de los planes y programas de la reforma.

En los inicios del ProForDEMS, en 2008, este se integra por un Diplomado en Competencias docentes en el Nivel Medio Superior, impartido a través de 42 Instituciones de Educación Superior (IES) y coordinado por la ANUIES, además de un conjunto de siete especialidades ofertadas en línea por la Universidad Pedagógica Nacional (UPN), las cuales fueron: Competencias docentes para la Educación Media Superior, Educación centrada en el aprendizaje, Aprendizaje y práctica docente en contextos multiculturales, Matemáticas y Tecnología, Competencias comunicativas, Enseñanza y aprendizaje de la historia y la ciencia y Aprendizaje de las ciencias. Las expectativas de la SEP (2008e), en ese momento, eran bastante optimistas, puesto que pretendían capacitar a 20 mil profesores en tal año, sumar 60 mil más en 2009 e incorporar a los 160 mil restantes en los dos años subsecuentes, para culminar con el total de 240 mil docentes que integraban la planta magisterial del bachillerato, meta que estuvo lejos de ser cumplida, puesto que para 2013, apenas se había alcanzado a capacitar a $56.2 \%$ de los docentes, de acuerdo con el $2^{\circ}$ Informe de Gobierno del Presidente Peña Nieto, según hemos expuesto antes. En el ciclo escolar 2013-2014, la SEMS establece los convenios correspondientes para continuar con el proceso de capacitación docente, donde participan la ANUIES, la Universidad Autónoma de Yucatán (UADY), la Universidad Autónoma de Colima (UCOL) y la Universidad Autónoma de 
Guanajuato (UGTO); mientras que en las más recientes convocatorias al respecto, se ofertan dos diplomados: La diversidad y la Educación Media Superior, impartido en línea por la Universidad Pedagógica Nacional del estado de Chihuahua (UPNECH) y el Diplomado de competencias docentes para el Nivel Medio Superior, desarrollado por la Benemérita Universidad Autónoma de Puebla (BUAP), la Universidad Autónoma del Estado de México (UAEM), la Universidad de Guadalajara (UdeG), la Universidad Autónoma de Sinaloa (UAS) y la Universidad de Colina (UCOL), de acuerdo con el análisis de Andrés Lozano Medina (2015).

En el apartado relativo al ProForDEMS, la presentación de la SEMS al Consejo Nacional de Autoridades Educativas, de septiembre de 2013, respecto de los "Avances y resultados en la Educación Media Superior”, informa que del total de 180852 profesores de las instituciones públicas del nivel, solo $62.1 \%$ se incorpora a algún programa de formación docente, de los cuales alrededor de $40 \%$ lo ha concluido.

En una valoración preliminar del mecanismo de gestión respecto del desarrollo de la planta docente, en la RIEMS y, quizás a manera de hipótesis de trabajo, tanto en el aspecto de la capacitación de los profesores, como en su proceso de certificación, bien podemos adelantar que su instrumentación ha estado caracterizada por dos principales perspectivas, estas son: por un lado, el predominio del interés de las autoridades educativas, y también de las instancias involucradas -la ANUIES, la UPN y las Universidades locales-, por la cantidad de docentes participantes, más que por la calidad y la pertinencia de la oferta y de las acciones implementadas; y por otro, la evidente despreocupación por el impacto real que han tenido estos dispositivos de profesionalización docente, en la realización de las prácticas educativas del nivel. Lejos de ello, persiste la ausencia de datos precisos y testables sobre los verdaderos logros del avance del SNB, la aplicación del MCC, la inversión en el fortalecimiento de la infraestructura institucional y el desarrollo de la formación profesional docente; por lo que respecta a las posibles transformaciones de las estrategias formativas, su impacto en el logro de los alumnos, el comportamiento de los índices de abandono escolar y la calidad del servicio educativo, al respecto de lo que existe un completo silencio por 
parte de las autoridades educativas federales, como ya previene la ASF "la SEP no formuló indicadores y metas de cobertura relativos a la atención a los docentes de las instituciones públicas de educación media superior con cursos de formación y actualización, ni para medir la contribución del programa en el logro educativo de los alumnos de Educación media superior" (2011: 6).

Por su parte, en lo general, las IES participantes solo se limitan a impartir los cursos aprobados por el Comité Directivo del SNB -con el uso de los recursos económicos correspondientes-, sin efectuar un seguimiento sistemático de los posibles efectos que generan en las prácticas docentes de los profesores atendidos; los investigadores educativos, por su parte, solo se han concretado a cuestionar el carácter neoliberal de la reforma, la intromisión de las autoridades federales en los planteles y subsistemas del nivel, además de la presunta naturaleza técnico-mecanicista del enfoque educativo por competencias. De ahí, pues, la importancia de preguntarse: ¿cuál es el impacto de la profesionalización docente en las prácticas educativas de la EMS?

\section{Implicaciones profesionales de las competencias docentes}

En la intención de definir el "perfil deseable del docente" de la EMS que posibilite trascender "los propósitos exclusivamente disciplinares y apoyen de manera integral a la formación de los jóvenes, así como la instauración de mecanismos y lineamientos sistemáticos con base a criterios claros para la certificación de competencias docentes que contribuyan a conformar una planta académica de calidad", según se propone en el Acuerdo 447 (2008d: 1), además de que sirvan de referente central para determinar los criterios, parámetros y estándares de ingreso, promoción y permanencia de los profesores de este nivel educativo -hecho que se omite en el citado Acuerdo-, la RIEMS dispone ocho competencias docentes, con sus correspondientes atributos.

Ahora bien, en relación con estas competencias, antes de plantear cualquier consideración profesional posible, resulta necesario establecer una distinción práctico-formal entre lo que delimita la enunciación de las competencias y los rasgos propios que determinan la expresión de sus atributos, en la inteligencia de 
que ninguno de ellos puede exceder lógica y ontológicamente los límites de cualificación de aquellas. Así, entonces, tenemos que la primera competencia se refiere a la autogestión formativa del profesor en cuanto profesional de la docencia -no como profesionista de su campo disciplinario (ver Cuadro 2)-; mientras que las competencias 2, 3, 4, 5 y 6 se remiten, de manera directa, a los ámbitos propios de la planeación docente, la gestión formativa y la evaluación educativa; en tanto que la séptima y la octava se encauzan hacia la conformación de un ambiente sano y participativo que propicie el desarrollo integral de los alumnos.

$\mathrm{Al}$ respecto es posible señalar tres principales incongruencias de competitividad docente: primera, tanto para cumplir con el propósito de la RIEMS de que los profesores participen en la toma de decisiones de la reforma y en el desarrollo de las competencias del perfil de egreso del MCC, como para que dispongan de las competencias necesarias de la planeación docente, requieren de una sólida competencia de análisis curricular, lo cual no es ningún atributo particular de las ocho competencias enunciadas; segunda, el modelo educativo que se propone es el enfoque de la formación por competencias, pero se plantea que el docente debe "facilitar, planear, constituir ambientes y evaluar procesos de aprendizaje", lo que resulta tan inconsistente como pretender fundamentar prácticas docentes de corte constructivista, recurriendo a los dispositivos pavlovianos de estímulo-respuesta; y tercera, la reforma propone una estrecha relación de la EMS con los sectores productivos y de la Educación Superior - punto nodal del insistente cuestionamiento de sus principales detractores-, pero el análisis de las ocho competencias docentes muestra que se dirigen, de manera preponderante, a las condicionantes internas del contexto escolar; en otras palabras, resulta necesaria una competencia adicional que posibilite al profesor de este nivel educativo, comprender la realidad socio-política y económico-cultural del entorno actual, tanto para contextuar sus prácticas formativas, como para responder a las condiciones psicosociales de los adolescentes del siglo XXI. En esta perspectiva, la Organización de los Estados Iberoamericanos (OEI, 2010) señala que tres competencias cuyo dominio por los actuales profesores, y en especial por los nuevos docentes, va a ser una de las claves del cambio educativo, son: 
Preparación para enseñar en la diversidad de contextos, culturas y alumnos. Capacidad para incorporar al alumnado en la sociedad del conocimiento. Disposición para educar en una ciudadanía multicultural, democrática y solidaria.(OEI, 2010, 136).

Sin embargo, en un momento histórico en que se demanda la apertura de la escuela a la realidad de los contextos locales, nacionales y globales, la RIEMS propone el perfil de un docente que piensa y actúa para la interioridad de la escuela. La falta de pertinencia educativa, allende las deficiencias curriculares, formativas, de apoyo y de infraestructura institucional que reconocen los promotores de la reforma, se debe más al carácter endogámico y autorreferencial de los sistemas escolares modernos. En efecto, la perspectiva y práctica didáctico-pedagógica que promueve la escuela moderna, reconoce al fenómeno educativo como si ocurriera en una suerte de limbo, esto es, al margen de toda determinación sociopolítica; mientras que la escuela contemporánea propone una educación abierta al devenir de las prácticas socioculturales y político-económicas de su contexto.

En relación con el conjunto de atributos definido por el Acuerdo 447, conviene advertir que excede el perfil profesional delimitado por las ocho competencias propuestas, al menos en los siguientes aspectos: por un lado, prefigura el viejo modelo del docente-investigador, promovido durante la década de los ochenta del siglo XX, y abandonado después, a causa de su inconsistencia epistemológico-profesional, puesto que el ser docente se legitima por sí mismo, sin necesidad de validar ser investigador ("Reflexiona e investiga sobre la enseñanza y sus propios procesos de construcción del conocimiento", se afirma desde el primer atributo, en SEP, 2008d: 2); por otro, demanda de un dominio pleno del uso, aplicación y orientación de las TIC, como si se tratase solo de un simple recurso instrumental de la docencia, cuando, en realidad, implica una cierta racionalidad distinta para pensar, gestionar e intervenir en las prácticas educativas, a partir de lo cual se requieren competencias profesionales más amplias que el esquema del magisterio tradicional, como bien lo demuestra el limitado alcance de Enciclomedia, en el proyecto de la Educación Básica del gobierno del presidente Fox, durante el 
sexenio 2000-2006 ("el programa no mejoró significativamente el desempeño educativo de sus educandos”, según determina la ASF, en 2008); y por último, proyecta la imagen de un auténtico "interventor educativo", con competencias específicas para identificar situaciones emergentes de mejora o potenciación de los procesos de formación, diseñar proyectos de transformación significativa, construir sinergias sociales, realizar gestión socioinstitucional y ponderar sus resultados efectivos, en el contexto escolar-de manera particular en el conglomerado de atributos de las competencias séptima y octava-, cuyas disposiciones profesionales trascienden las competencias docentes de cualquier profesor en el Sistema Educativo, y con mayor razón, las enunciadas para el perfil profesional docente decidido por la reforma. Al respecto, resulta pertinente señalar que la escuela contemporánea no se reduce solo a la confluente actuación profesional de profesores y directivos, como parece entender la RIEMS, a través de los "mecanismos de gestión de la reforma"; por el contrario, la educación del siglo XXI se resuelve en la acción coordinada, integrada e interdisciplinaria de diversos tipos de profesionistas.

En torno a la conjunción descriptiva de las ocho competencias del perfil profesional docente de la reforma, y sus cuarenta ambiciosos atributos, es bien factible señalar los aspectos que a continuación se comentan, a saber: en principio, su definición discursivo-formal representa la declaración de un ideal educativo, pero descontextuado de la realidad concreta que prevalece en el bachillerato, irónicamente expuesta en los documentos de legitimación de la necesidad impostergable de implementar la propia RIEMS, pero soslayada al momento de decidir los rasgos docentes; esto es, si no existen instituciones de formación inicial para los maestros del nivel, las acciones de actualización precedentes han sido aisladas, insuficientes y carentes de pertinencia, además de que el posgrado en este campo es todavía incipiente, no resulta demasiado temerario preguntarse: ¿cómo, entonces, podrán desarrollar estas competencias los profesores y técnicos docentes de la EMS?, una vez que ha quedo claro que carecen de cualquier fortalecimiento didáctico-pedagógico antecedente; la respuesta parece pronta y fácil: el ProforDEMS que se instrumenta desde la UPN, la ANUIES y sus IES afiliadas. 
Sin embargo, el asunto no se resuelve para los aspirantes a ingresar como profesionales docentes del bachillerato, porque este programa se dirige a los profesores en servicio, ¿cómo y dónde, pues, se formarán las nuevas generaciones de maestros para el bachillerato?; aun cuando también es conveniente percatarse que el marco de desarrollo del perfil profesional docente, dispuesto por el Acuerdo 447, es tan amplio y diverso que no puede alcanzarse mediante la instrumentación de simples estrategias de actualización y capacitación emergente, de corto y mediano plazo, como lo ha realizado el programa; todo lo contrario, en realidad, su dimensión alcanza para determinar el perfil de egreso de un verdadero proceso de formación a nivel de posgrado, o en su defecto, un proyecto sistemático de formación continua, de largo plazo, dirigido expresamente a los maestros de la EMS. No puede obviarse que el desarrollo de las ocho competencias y sus correspondientes atributos desborda los propósitos planteados por el ProForDEMS, a partir de lo que se hace necesario diseñar un amplio programa de formación magisterial de segundo orden. Aunque es indispensable considerar que las acciones de certificación docente instrumentadas por la CERTIDEMS y su impacto en las posibilidades concretas del incremento de horas, la promoción y la permanencia laboral han generado en el imaginario de la comunidad docente del bachillerato la arraigada convicción de que resulta ocioso e improductivo suscribirse a un postgrado, porque parece representar una opción más tangible incorporarse y sostenerse en los cursos de capacitación emergente de la reforma, ya que les brinda una aparente garantía de obtener una certificación más expedita y, por ende, mejores oportunidades laborales en el nivel, según declaran de manera expresa distintos profesores del bachillerato, cuando se les interroga respecto de su falta de interés por incorporarse a los estudios de postgrado.

En cuanto a la relación entre las competencias del perfil profesional docente y las competencias genéricas del desempeño terminal compartido, que constituyen el perfil de egreso de la EMS, pueden apreciarse con mayor claridad en el siguiente cuadro: 


\section{CUADRO 2. Relación de las Competencias Docentes con las competencias genéricas del MCC}

\begin{tabular}{|c|c|c|}
\hline No. & Competencias Docentes & Competencias Genéricas \\
\hline 1. & $\begin{array}{l}\text { Organiza su formación continua a lo largo de su } \\
\text { trayectoria profesional. }\end{array}$ & \\
\hline 2. & $\begin{array}{l}\text { Domina y estructura los saberes para facilitar } \\
\text { experiencias de aprendizaje significativo. }\end{array}$ & \\
\hline 3. & $\begin{array}{l}\text { Planifica los procesos de enseñanza y de } \\
\text { aprendizaje atendiendo al enfoque por } \\
\text { competencias, y los ubica en contextos } \\
\text { disciplinares, curriculares y sociales amplios. }\end{array}$ & \\
\hline 4. & $\begin{array}{l}\text { Lleva a la práctica procesos de enseñanza } \\
\text { y de aprendizaje de manera efectiva, creativa } \\
\text { e innovadora a su contexto institucional. }\end{array}$ & \\
\hline 5. & $\begin{array}{l}\text { Evalúa los procesos de enseñanza y de } \\
\text { aprendizaje con un enfoque formativo. }\end{array}$ & \\
\hline \multirow{3}{*}{6.} & \multirow{3}{*}{$\begin{array}{l}\text { Construye ambientes para el aprendizaje } \\
\text { autónomo y colaborativo. }\end{array}$} & $\begin{array}{l}\text { 1. Se conoce y valora a sí mismo y aborda problemas y } \\
\text { retos teniendo en cuenta los objetivos que persigue. }\end{array}$ \\
\hline & & $\begin{array}{l}\text { 2. Es sensible al arte y participa en la apreciación e } \\
\text { interpretación de sus expresiones en distintos géneros. }\end{array}$ \\
\hline & & $\begin{array}{l}\text { 7. Aprende por iniciativa e interés propio a lo largo de la } \\
\text { vida. }\end{array}$ \\
\hline \multirow{4}{*}{7.} & \multirow{4}{*}{$\begin{array}{l}\text { Contribuye a la generación de un ambiente } \\
\text { que facilite el desarrollo sano e integral de los } \\
\text { estudiantes. }\end{array}$} & 3. Elige y practica estilos de vida saludables. \\
\hline & & $\begin{array}{l}\text { 6. Sustenta una postura personal sobre temas de interés y } \\
\text { relevancia general, considerando otros puntos de vista } \\
\text { de manera crítica y reflexiva. }\end{array}$ \\
\hline & & $\begin{array}{l}\text { 9. Participa y colabora de manera efectiva en equipos } \\
\text { diversos. }\end{array}$ \\
\hline & & $\begin{array}{l}\text { 10. Mantiene una actitud respetuosa hacia la } \\
\text { interculturalidad y la diversidad de creencias, valores, } \\
\text { ideas y prácticas sociales. }\end{array}$ \\
\hline 8. & $\begin{array}{l}\text { Participa en los proyectos de mejora continua de } \\
\text { su escuela y apoya la gestión institucional. }\end{array}$ & \\
\hline
\end{tabular}

Fuente: Elaboración Propia

Las competencias genéricas que pueden relacionarse con las competencias del perfil profesional del profesor de bachillerato, con respecto a la planeación docente, la gestión formativa y la evaluación educativa, son: 
4. Escucha, interpreta y emite mensajes pertinentes en distintos contextos mediante la utilización de medios, códigos y herramientas apropiados.

5. Desarrolla innovaciones y propone soluciones a problemas a partir de métodos establecidos.

Por su parte, las competencias genéricas que trascienden el marco de atribución de las competencias docentes del profesor de EMS, definidas por el Acuerdo 447, en el entendido que el principal problema de la educación moderna es precisamente la falta de relación entre el desarrollo formativo de la escuela -solo pertinente para las necesidades y exigencias académicas del propio sistema escolar-, y las demandas socioambientales, políticoculturales y ético-económicas, son:

9. Participa con una conciencia cívica y ética en la vida de su comunidad, región, México y el mundo.

11. Contribuye al desarrollo sustentable de manera crítica, con acciones responsables.

Al final del análisis de los distintos principios, propósitos y componentes centrales de la RIEMS, más allá del estridentismo estadístico oficial y los recursos invertidos en el ramo, es posible reconocer que el desarrollo de la planta docente, en sí mismo, no constituye una prioridad para la propia reforma ni para las autoridades educativas, ante la política intención de instaurar dispositivos de control institucional sobre los subsistemas, modalidades, planteles y profesores que participan del bachillerato, en México -conforme a la agenda convenida con el BID y la OCDE, entre otros; siguiendo los planteamientos de Manuel Pérez Rocha, quien señala que mediante la RIEMS (...), la SEP hace un nuevo intento de controlar ese nivel educativo y orientarlos de conformidad con la ideología y perspectivas del gobierno federal (2011, s/p)-, pues, además de constituir un mecanismo secundario de su gestión y de obviar la necesidad de realizar un seguimiento sistemático del impacto real de las acciones emergentes de capacitación de los profesores en las prácticas educativas del nivel, cierto es que el mismo ProForDEMS se ha venido diluyendo ante el cre- 
ciente protagonismo del Comité directivo del SNB, la reiteración de las acciones y modalidades emprendidas desde el principio por las diversas instancias participantes en el programa -verbigracia, la ANUIES prosigue ofertando el mismo Diplomado en competencias docentes del nivel medio superior, alcanzando ya la séptima generación, y la UPN continúa con la misma estrategia de los diplomados y cursos en línea-, sin que exista un interés genuino por diseñar un programa sistemático de largo plazo, que desarrolle, de manera efectiva, las ocho competencias del perfil profesional docente definido por la RIEMS, pese a sus evidentes insuficiencias y contrasentidos. De hecho, conforme a los datos expuestos en el presente análisis, es más que conveniente impulsar una profunda estrategia integral de congruencia formal y educativa de las diferentes propuestas de la reforma.

\section{REFERENCIAS BIBLIOGRÁFICAS}

Auditoría Superior de la Federación. "Auditoría de Desempeño: 12-0-11100-07-0383. SEP. Equipamiento de Escuelas de Educación Básica”, México, ASF, 2008.

Auditoría Superior de la Federación. "Auditoría de Desempeño: 11-0-111000-07-0399. SEP. Formación de Docentes de la Educación Media Superior”, México, ASF, 2011.

Auditoría Superior de la Federación. "Evaluación Número 290. Educación Superior”, México, ASF, 2012.

Banco Interamericano de Desarrollo. "México: Retos para el Sistema Educativo 2012-2018”, México, BID, 2012.

Conferencia Regional de la Educación Superior. "Declaración de la Conferencia Regional de la Educación Superior en América Latina y el Caribe. Declaraciones y Plan de Acción. CRES 2008”, en Perfiles Educativos, vol. XXXI, núm. 125, México, IISUE, UNAM, 2009, pp. 90-108

Diario Oficial de la Federación. "Programa Sectorial de Educación 2013-2018", en DOF, México, 2013 a.

Diario Oficial de la Federación. "Ley General del Servicio Profesional Docente", en DOF, México, 2013b.

Ezpeleta, Justa et al. "NO a la Reforma Curricular Improvisada", en Odiseo, Revista Electrónica de Pedagogía, 2008, Disponi- 
ble en http://www.odiseo.com.mx/marcatexto/no-reformacurricular-improvisada. Fecha de acceso, 2 de agosto de 2016. Instituto Nacional de Evaluación Educativa. "Cálculos con Base en las Estadísticas Continuas del Formato 911”, México, SEP-Dirección General de Profesiones, 2011.

Instituto Nacional de Evaluación Educativa. "Panorama Educativo de México, 2013. Indicadores del Sistema Educativo Nacional. Educación Básica y Media Superior”, México, INEE, 2014.

Instituto Nacional de Evaluación Educativa. "Lineamientos para llevar a cabo la Evaluación del Desempeńo del Personal Docente, Técnico Docente y de quienes Ejerzan Funciones de Dirección y Supervisión en Educación Básica y Media Superior en el Ciclo Escolar 2016-2017”, en DOF, México, 2016.

Lozano Medina, Andrés. "La RIEMS y la Formación de los Docentes de la Educación Media superior en México", en Perfiles Educativos, vol. XXXVII, México, IISUE, UNAM, 2015, pp. 108-124.

Organización de Estados Iberoamericanos. Metas Educativas 2021. La educación que queremos para la generación de los Bicentenarios, OEI/CEPAL, 2010.

Organización para la Cooperación y el Desarrollo Económicos. "Acuerdo de Cooperación México-OCDE para Mejorar la Calidad de la Educación de las Escuelas Mexicanas. Mejorar las Escuelas: Estrategias para la Acción en México. Establecimiento de un Marco para la Evaluación e Incentivos para Docentes: Consideraciones para México. Resúmenes Ejecutivos", OCDE, México, 2010.

Organización para la Cooperación y el Desarrollo Económicos. Perspectivas de la Educación Media Superior (a Finales de Sexenio), 2011. Disponible en: http://archivo.estepais.com/ site/2011/perspectivas-de-la-educacion-media-superior-afinales-de-sexenio/. Fecha de acceso, 30 de julio de 2016.

Pérez Rocha, Manuel. "Las Humanidades y la Barbarie de la RIEMS”, en: La Jornada en Linea, México, 2011. Disponible en http://www.jornada.unam.mx/2011/06/16/politica /020a2pol. Fecha de acceso, 16 de diciembre de 2016.

Poder Ejecutivo Federal. "Plan Nacional de Desarrollo 2007-2012". Gobierno de los Estados Unidos Mexicanos, México, 2007. 
Secretaría de Educación Pública. "Programa Sectorial de Educación 2007-2012”, México, SEP, 2007.

Secretaría de Educación Pública. "Acuerdo Número 442 por el que se Establece el Sistema Nacional de Bachillerato en un Marco de Diversidad", México, SEP, 2008a.

Secretaría de Educación Pública. "Reforma Integral de la Educación Media Superior en México: La Creación de un Sistema Nacional de Bachillerato en un Marco de Diversidad", México, SEP, 2008b.

Secretaría de Educación Pública. "Acuerdo Número 444 por el que se Establecen las Competencias que Constituyen el Marco Curricular Común del Sistema Nacional del Bachillerato", México, DOF, 2008c.

Secretaría de Educación Pública. "Acuerdo Número 447 por el que se Establecen las Competencias Docentes para Quienes Impartan Educación Media Superior en la Modalidad Escolarizada”, México, DOF, 2008d.

Secretaría de Educación Pública. "Publica la SEP, Convocatoria para Formación de Docentes en Educación Media Superior", 2008e. Disponible en: http://www.sems.gob.mx/ es_mx/sems/Port_publica_la_sep_convocatoria_para_ formacion_de. Fecha de acceso, 3 de agosto de 2016.

Secretaría de Educación Pública. "Principales Cifras del Sistema Educativo Nacional 2012-2013", México, SEP, 2013 a.

Secretaría de Educación Pública. "Antecedentes de la RIEMS", México, SEP, 2013b. Disponible en: http://www.sems. gob.mx/es_mx/sems/antecedentes_dgb. Fecha de acceso, 27 de julio de 2016.

Secretaría de Educación Pública. "Avances y Resultados en la Educación Media Superior”, México, SEP, 2013c. Disponible en: http://docplayer.es/8938102-Avances-y-resultados-en-la-educacion-media-superior.html. Fecha de acceso, 5 de agosto de 2016.

Secretaría de Educación Pública. "E007 Servicios de Educación Media Superior. Diagnóstico Inicial”, México, SEP, 2015. Secretaría de Educación Pública. "Evaluación del Desempeño Docente y Técnico Docente. Perfil, Parámetros e Indicadores para Docentes y Técnicos Docentes en Educación Superior”, México, SEP, 2016. 